\title{
Effect of thermal radiation on MHD flow of blood and heat transfer in a permeable capillary in stretching motion
}

\author{
J. C. Misra • A. Sinha
}

Received: 4 March 2012/Accepted: 13 December 2012/Published online: 3 March 2013

(C) The Author(s) 2013. This article is published with open access at Springerlink.com

\begin{abstract}
In this paper, a theoretical analysis is presented for magnetohydrodynamic flow of blood in a capillary, its lumen being porous and wall permeable. The unsteadiness in the flow and temperature fields is caused by the timedependence of the stretching velocity and the surface temperature. Thermal radiation, velocity slip and thermal slip conditions are taken into account. In order to study the flow field as well as the temperature field, the problem is formulated as a boundary value problem consisting of a system of nonlinear coupled partial differential equations. The problem is analysed by using similarity transformation and boundary layer approximation. Solution of the problem is achieved by developing a suitable numerical method and using high speed computers. Computational results for the variation in velocity, temperature, skin-friction co-efficient and Nusselt number are presented in graphical/tabular form. Effects of different parameters are adequately discussed. Since the study takes care of thermal radiation in blood flow, the results reported here are likely to have an important bearing on the therapeutic procedure of hyperthermia, particularly in understanding/regulating blood flow and heat transfer in capillaries.
\end{abstract}

J.C. Misra was earlier a Professor at the Department of Mathematics at Indian Institute of Technology, Kharagpur, India.

J. C. Misra ( $\square)$

Department of Mathematics, Institute of Technical Education

and Research, Siksha O Anusandhan University,

Bhubaneswar, India

e-mail: misrajc@gmail.com

A. Sinha

School of Medical Science and Technology,

Indian Institute of Technology, Kharagpur 721302, India

\section{List of symbols}

$\eta \quad$ Dimensionless distance

$u \quad$ Velocity of blood along the axis of the capillary

$v \quad$ Transverse velocity of blood in the capillary

$T \quad$ Temperature of blood at any point in the capillary

$u_{s} \quad$ Velocity-slip at the wall

$U_{w} \quad$ Stretching velocity

$V_{w} \quad$ Injection/suction velocity

$T_{w} \quad$ Surface temperature

$T_{s} \quad$ Thermal slip

$B(t)$ Time-dependent magnetic field intensity

$k_{1}(t)$ Time-dependent permeability parameter of blood

$k_{2} \quad$ Constant permeability of the medium

$k_{3} \quad$ Non-dimensional permeability parameter

$q_{r} \quad$ Radiative heat flux of blood

$k \quad$ Thermal conductivity of blood

$N \quad$ Velocity-slip factor

$K \quad$ Thermal-slip factor

$M \quad$ Hartmann number of the blood mass

A Unsteadiness parameter

$\mathrm{Nr} \quad$ Radiation parameter

$S_{f} \quad$ Non-dimensional velocity-slip factor

$S_{t} \quad$ Non-dimensional thermal-slip factor

$\mathrm{Pr} \quad$ Prandtl number

$S \quad$ Injection/Suction parameter

$\rho \quad$ Density of blood

$\sigma \quad$ Electrical conductivity of blood

$v \quad$ Kinematic coefficient of viscosity of blood

$c_{p} \quad$ Specific heat at constant pressure

$\sigma^{\star} \quad$ Stefan-Boltzmann constant

$k^{\star} \quad$ Mean absorption co-efficient

$C_{f} \quad$ Skin-friction coefficient

$N u_{x} \quad$ Local Nusselt number 


\section{Introduction}

Radiation effect in blood flow is an important subject of research, because it has got significant applications in Biomedical engineering and several medical treatment methods, particularly in thermal therapeutic procedures. Infrared radiation is one of the frequently used techniques for making heat treatment to various parts of the human body. This technique is preferred in heat therapy, because by using infrared radiation it is possible to directly heat the blood capillaries of the affected areas of the body. In the treatment of muscle spams, myalgia (muscle pain), chronic wide-spreed pain (fibromyalgia, in medical terms) and permanent shortening of muscle (medically called as contracture), heat therapy is found to be very effective. It is also used in the treatment of bursitis, that is, inflammation of the fluid-filled sac (bursa) that lies between tendon and bone, or between tendon and skin. Several experimental investigations have been carried out by some researchers (cf. Kobu [1], Inoue and Kabaya [2], Nishimoto et al. [3]) to examine the effects of infrared radiation/ultrasonic radiation on blood flow. The effect of radiative heat transfer on blood flow in a stenosed artery was studied theoretically by Prakash and Makinde [4]. By using a numerical model, He et al. [5] discussed the effect of temperature on blood flow in human breast tumor under laser irradiation.

While flowing through the arterial tree, blood carries a large quantity of heat to different parts of the body. On the skin surface, the transfer of heat can take place by any of the four processes: radiation, evaporation, conduction and convection. It is known that in the case of radiative heat transfer, energy is transferred through space by means of electromagnetic wave propagation. There are several determinants for the quantity of heat that blood can carry with it, namely, (1) heat transfer coefficient of blood, (2) density of blood, (3) velocity of blood flow, (4) radius of the artery and (5) temperature of the tissues that surround the artery. Out of these, since Reynolds number is related to the velocity and density of blood as well as the arterial radius, the quantity of heat carried by blood can be regarded as dependent only on Reynolds number and heat transfer coefficient of blood, and the temperature of the tissues surrounding the artery.

It may further be mentioned that blood flow enhances when a man performs hard physical work and also when the body is exposed to excessive heat environment. In cases like these, blood circulation can not remain normal. In order to take care of the increase in blood flow, the dimensions of the artery have to increase suitably. It is known that when the temperature of the surroundings exceeds $20{ }^{\circ} \mathrm{C}$, heat transfer takes place from the surface of the skin by the process of evaporation through sweating and when the temperature is below $20^{\circ} \mathrm{C}$, the human body loses heat by conduction and radiation both. Blood flow with radiative heat transfer was discussed by Ogulu and Bestman [6] on the basis of a theoretical study. Shah et al. [7] designed an instrument for measuring the heat convection coefficient on the endothelial surface of arteries. Effects of pulsatile blood flow in arteries during thermal therapy were studied by Craciunescu and Clegg [8] as well as by Horng et al. [9]. Shrivastava and Roemer [10] studied heat transfer rate from one blood vessel to another and also that from a blood vessel to tissue.

In addition to the aforementioned usefulness of radiative heat transfer in blood vessels, the radiation effect in blood is of significant interest to clinicians in the therapeutic procedure of hyperthermia, which has a well-recognized effect in oncology. Its effect is achieved by overheating the cancerous tissues (cf. Szasz [11]) by means of electromagnetic radiation. Misra et al. [12] reported theoretical estimates of blood flow in arteries during the therapeutic procedure of electromagnetic hyperthermia used for cancer treatment. Some relevant useful discussions are also available in that paper. Different aspects of hyperthermia were discussed/studied by several researchers (cf. Abe and Hiraoka [13], Molls [14], Chen and Roemer [15], Overgaard [16], Feldmann et al. [17], Habash et al. [18]). Deng et al. [19] observed significant thermal effects in blood vessels during cryosurgery by means of infrared temperature mapping.

Two major functions that blood performs while flowing in the circulatory system are to carry nutrients and to supply heat to body tissues. The exchange of materials mainly takes place at the capillary level. However, there exists evidence to support that materials are also transported across the permeable walls of arteries (and veins) [20]. The transport of water across the arterial wall is of interest in the study of metabolism and pathology of an artery. The nourishment of the arterial wall depends predominantly on the transport of materials from the arterial lumen. This transport can, sometimes, cause the genesis and the progression of arterial diseases such as atherosclerosis, atherogenesis, atheroma. Oka and Murata [21] discussed the steady flow of blood through a permeable capillary wall. Pallat et al. [22] studied the flow field in an infinite permeable tube.

Computational bio-fluid dynamics has made it possible to simulate complex flows in vascular passages such as cerebral and carotid arteries. The geometry of blood vessels and arterial walls, their structure and mechanical properties are largely dependent on pressure and flow conditions. Moreover, the effect of porosity of the medium on blood flow can not be ignored in many pathological situations. Vankan et al. [23] performed a simulation for blood flow through a contracting muscle, with a hierarchical structure 
of pores. Dash et al. [24] employed the Brinkman equation to model the pathological blood flow when there is accumulation of fatty plaques of cholesterol in the lumen of an arterial segment and artery-clogging takes place by blood clots. They treated the clogged region as a porous medium and assumed the permeability parameter to be either a constant or varying in the radial direction. Preziosi and Farina [25] analyzed flow of a Newtonian fluid in a porous medium in the presence of mass exchange between the constituents.

The magnetic response of blood involves paramagnetic contribution emerging from the iron content of hemoglobin molecules and the diamagnetic contribution emerging from hydrogen, oxygen, nitrogen and carbon atoms contained in vessel tissues. The red blood cell (erythrocyte) is a major biomagnetic substance and therefore, it is quite possible that blood flow will be influenced by the presence of an external magnetic field. Reduction in blood flow rate is caused by either an increase in flow resistance or a reduction in blood pressure.

The effect of a magnetic field on blood flow has been analyzed theoretically by Chen [26] treating blood as an electrically conducting fluid. Tzirtzilakis and Tanoudis [27] studied the biomagnetic fluid flow over a stretching sheet. Pulsed magnetic fields have been used to treat various conditions, such as soft-tissue injury [28], chronic pelvic pain [29]. It has also been reported that application of a magnetic field is useful for nerve regeneration [30, 31], bone grafts [32] and fracture healing [33]. Keeping all these in mind, presence of an external magnetic field has been paid due consideration in the present study.

The flow over a stretching surface has received attention of several researchers in the past. Flow and heat transfer problems for a stretching sheet in different situations were studied by Liu [34] as well as by Sajid and Hayat [35]. However, in these studies, the flow and temperature fields were considered to be steady. But the cases in which the stretching force and surface temperature vary with time, have received scant attention of previous researchers. One of the merits of the present study is that it takes due care of the time-dependence of the stretching force and surface temperature of blood capillaries. This makes the study closer to the real situation.

It is a well known fact that a viscous fluid normally sticks to a boundary, i.e., there is no slip of the fluid relative to the boundary. However, Beavers and Joseph [36] observed that there are many situations, where there may be a partial slip between the fluid and the boundary. For many fluids, e.g. particulate fluids, although the motion is governed by Navier-Stokes equations, it is desirable that the no-slip condition at the boundary should be replaced by velocity-slip condition. Nubar [37] suggested the presence of a red cell (erythrocyte) slip at the vessel wall. Misra et al. [38] conducted a study concerning blood flow through an arterial segment where they considered no-slip condition at the vessel wall. Subsequently however, Misra and Shit [39] investigated the role of slip velocity in blood flow through stenosed arteries.

Since blood is an electrically conducting fluid, in the presence of a magnetic field, its flow exhibits magnetohydrodynamic (MHD) behaviour. Pal, Misra and Gupta [40] investigated the steady MHD flow of an electrically conducting fluid (with particular reference to blood flow in arteries) in a slowly varying channel in the presence of a uniform transverse magnetic field. Misra and Shit [41] developed a mathematical model of the flow of a biomagnetic viscoelastic fluid over a stretching sheet. Misra and Shit [42] also investigated the flow and heat transfer of an MHD viscoelastic fluid in a channel with stretching walls. All these investigations carried out by Misra and his research group have been recognized as benchmark contributions in the field of physiological fluid dynamics.

In view of the discussion made above, we have been motivated towards investigating the problem of unsteady magnetohydrodynamic flow and heat transfer of blood in a narrow permeable blood vessel, which is in a state of unsteady stretching motion. The analysis of the model takes due care of thermal radiation that takes place when blood flows inside the vessel. Also, the thermal slip accompanied by velocity-slip at the capillary wall has been accounted for in the study. Because of the permeability of capillary blood vessel walls, consideration of the slip-velocity at the wall makes the study closer to the reality. Moreover, magnetic effects of blood (as discussed earlier) on the flow and heat transfer of blood have been considered under the purview of the same model. Keeping in view that during surgery, or thermal therapy, the clinician needs to control the blood flow by regulating the intensity of the externally applied magnetic field which the system is subject to, has been taken to be timedependent. These are some of the novel features of the study. It may be mentioned here that although some attempts were made by previous researchers (cf. Raptis [43]) to study thermal radiation and free convection flow of a viscous fluid through a porous medium bounded by porous plates with constant suction velocity, the study was limited to steady flow in the absence of any magnetic field.

Moreover, keeping in the mind that in the physiological state, blood vessels (more particularly, the capillary blood vessels) execute a stretching motion, the blood vessel has been modelled as one that executes unsteady stretching motion and has its wall permeable, as per experimental reports available in scientific literatures.

The analysis of the model gives rise to a set of nonlinear partial differential equations. For solving these 
equations subject to the boundary conditions appropriate to the physical problem, we have made an endeavour to develop a suitable numerical method that involves the use of finite differences, together with the method of discretization, where the matrix of unknown physical variables is diagonally dominant. The computational results obtained by the use of high speed computers have been presented through graphs and tables. The results give the theoretical estimates of various parameters that govern the physical problem.

The present research being motivated towards studying the flow and heat transfer in capillary blood vessels bear the promise of useful applications in clinical sciences, particularly in thermal therapy. It also bears the potential of significant applications in different problems of biomedical engineering. In addition to these, being primarily a study on a problem of unsteady flow and heat transfer over a stretching sheet, it will find some important applications in various industrial manufacturing processes that involve interaction of the stretching sheet flow with radiative heat transfer. By using the theoretical analysis, changing the values of the parameters appropriately and employing the method of parametric variation, the estimates of different parameters controlling various problems of manufacturing industries can be obtained. The study is thus useful in assessing the quality of the final products too.

\section{The model}

Let us investigate the flow of blood through a narrow artery having permeable wall, which is in a state of unsteady stretching motion. We consider that the artery is under the action of a time-dependent magnetic field $B(t)$, which acts in a direction transverse to that of the flow. As per experimental observation, blood is a conducting fluid. Hence under the action of the magnetic field, the flow of blood will be of magneto-hydrodynamic (MHD) nature. In the analysis, presented in the next section, it is assumed that the magnetic Reynolds number is much less than unity so that the induced magnetic field is negligible in comparison to the applied magnetic field. Let us consider the axes of $x$ and $y$ in directions parallel and perpendicular to the axis of the artery (cf. Fig. 1). At time $t=0$, the blood vessel is considered to be impulsively stretched with velocity $U_{w}(x, t)$ along the $x$ - axis, where the origin is kept fixed in the fluid medium of ambient temperature $T_{\infty}$.

As mentioned in a very recent communication by Misra et al. [44], although the flow in the circulatory system is mainly three-dimensional, blood flow in micro-vessels can be taken to be two-dimensional in many cases.

Thus in the analysis that follows, blood flow in a narrow vessel will be approximated as a magneto-hydrodynamic

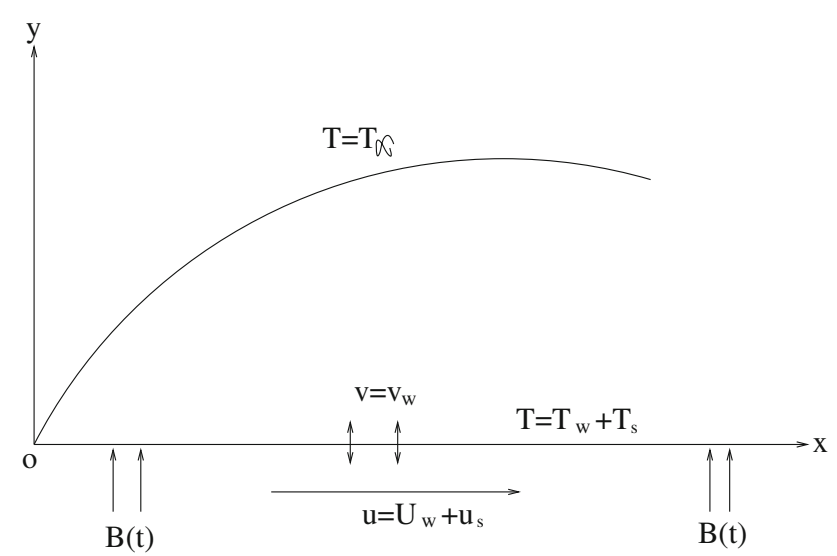

Fig. 1 Sketch of the physical problem

unsteady flow over a stretching sheet. The wall being permeable, velocity-slip as well as thermal slip will be taken into account. The associated heat transfer problem will also analysed. Consideration of the thermal radiation that takes place during blood flow in narrow vessels will also be made.

\section{Analysis}

The analysis of the present model will be carried out under the purview of all the considerations/assumptions made in Sect. 2, keeping in view the discussions made in Sect. 1. Thus the flow will be considered as a channel flow. Hence the equations that govern the flow of blood for the present problem may be considered in the form

$$
\begin{aligned}
& \frac{\partial u}{\partial t}+u \frac{\partial u}{\partial x}+v \frac{\partial u}{\partial y}=v \frac{\partial^{2} u}{\partial y^{2}}-\frac{\sigma B^{2}(t)}{\rho} u-\frac{v}{k_{1}(t)} u, \\
& \frac{\partial T}{\partial t}+u \frac{\partial T}{\partial x}+v \frac{\partial T}{\partial y}=\frac{k}{\rho c_{p}} \frac{\partial^{2} T}{\partial y^{2}}-\frac{1}{\rho c_{p}} \frac{\partial q_{r}}{\partial y}
\end{aligned}
$$

and

$$
\frac{\partial u}{\partial x}+\frac{\partial v}{\partial y}=0
$$

The definitions of all the symbols involved in the equations are included in the Nomenclature. The assumptions under which the above-written governing equations are valid, have been clearly stated in the formulation of the model, presented in Sect. 2. The boundary conditions for the problem can be written as (cf. $[45,46])$

$$
\begin{gathered}
u=U_{w}+N \mu \frac{\partial u}{\partial y}=U_{w}+u_{s}, \quad v=V_{w}, \\
T=T_{w}+K \frac{\partial T}{\partial y}=T_{w}+T_{s} \quad \text { at } \quad y=0
\end{gathered}
$$


and

$u \rightarrow 0, \quad T \rightarrow T_{\infty} \quad$ at $\quad y \rightarrow \infty$

In Eq. (4) $V_{w}$ represents the blood velocity at the capillary wall and is equal to injection/suction velocity given by

$V_{w}=-\sqrt{\frac{v U_{w}}{x}} f(0)$.

Equation (6) implies that the mass transfer at the surface of the capillary wall takes place with a velocity $V_{w}$, where $V_{w}>0$ in the case of injection and $V_{w}<0$ in the case of suction. In Eq. (1), $k_{1}(t)=k_{2}(1-c t)$ represents the timedependent permeability parameter, in Eq. (4), $N=N_{0}(1-$ $c t)^{\frac{1}{2}}$ is the velocity-slip factor, and $K=K_{0}(1-c t)^{\frac{1}{2}}$ is the thermal slip factor. The no-slip conditions can be recovered, by setting $N=K=0$. The velocity $U_{w}(x, t)$ of the stretching motion of the blood capillary and the surface temperature $T_{w}(x, t)$ are considered in the following forms

$U_{w}(x, t)=\frac{a x}{1-c t}, \quad$ and $\quad T_{w}(x, t)=T_{\infty}+\frac{b x}{1-c t}$,

where $a, b$ and $c$ are the constants such that $a>0, b \geq 0, c \geq 0$ and $c t<1$. Let us choose $B(t)=B_{0}(1-c t)^{-\frac{1}{2}}$, where $B_{0}$ is a constant representing the magnetic field strength at $t=0$. By using Rosseland approximation, the relative heat flux can be expressed as

$q_{r}=-\frac{4 \sigma^{\star}}{3 k^{\star}} \frac{\partial T^{4}}{\partial y}$

Considering that the temperature differences within the mass of blood inside the capillary are sufficiently small, using Taylor expansion and neglecting higher-order terms, we write:

$T^{4}=4 T_{\infty}^{3} T-3 T_{\infty}^{4}$

$T_{\infty}$ being the embedded temperature. We now introduce the following dimensionless functions $f$ and $\theta$, and similarity variable $\eta$ as

$\eta=\sqrt{\frac{U_{w}}{v x} y}, \quad f(\eta)=\psi / \sqrt{v x U_{w}} \quad$ and $\quad \theta=\frac{T-T_{\infty}}{T_{w}-T_{\infty}}$.

Now substituting (10) into the Eqs. (1) and (2), we get the following set of ordinary differential equations

$f^{\prime \prime \prime}+f f^{\prime \prime}-f^{\prime 2}-A\left(f^{\prime}+\frac{1}{2} \eta f^{\prime \prime}\right)-M^{2} f^{\prime}-\frac{1}{k_{3}} f^{\prime}=0$

and

$\frac{(1+N r)}{\operatorname{Pr}} \theta^{\prime \prime}+f \theta^{\prime}-\theta f^{\prime}-A\left(\theta+\frac{1}{2} \eta \theta^{\prime}\right)=0$, where $A, M, k_{3}, N r$ and $P r$ are non-dimensional parameters called respectively the unsteadiness parameter, Hartman number, permeability parameter, radiation parameter and Prandtl number given by $A=$ $\frac{c}{a}, M=B_{0} \sqrt{\frac{\sigma}{\rho a}}, k_{3}=\frac{a k_{2}}{v}, N r=\frac{16 \sigma^{\star} T_{\infty}^{3}}{3 k^{\star} k}$ and $\operatorname{Pr}=\frac{\mu c_{p}}{k}$. The boundary conditions (4) and (5) give rise to

$f(0)=S, \quad f^{\prime}(0)=1+S_{f} f^{\prime \prime}(0) \quad$ and

$\theta(0)=1+S_{t} \theta^{\prime}(0)$.

We further consider the following conditions:

$f^{\prime}(\eta) \rightarrow 0, \quad \theta(\eta) \rightarrow 0 \quad$ as $\quad \eta \rightarrow \infty$.

In Eq. (13), $S<0$ and $S>0$ correspond to injection and suction respectively. The non-dimensional velocity-slip factor $S_{f}$ and the non-dimensional thermal slip factor $S_{t}$ are defined by $S_{f}=N_{0} \rho \sqrt{a v}$ and $S_{t}=K_{0} \sqrt{\frac{b}{v}}$.

In the equations written above, primes denote derivatives with respect to $\eta$. It may be noted that if we set $A=0$ in Eqs. (11) and (12), the problem will be reduced to one in the steady state.

\section{Computational procedure: development of a suitable numerical method}

The two ordinary differential Eqs. (11) and (12) subject to the boundary conditions (13) and (14) constitute a nonlinear boundary value problem. To solve the boundary value problem, we have developed a suitable computational procedure, as outlined below. Writing $f^{\prime}=P$ in (11), we get

$P^{\prime \prime}+f P^{\prime}-P^{2}-A\left(P+\frac{1}{2} \eta P^{\prime}\right)-M^{2} P-\frac{1}{k_{3}} P=0$.

Also the boundary conditions (13) and (14) will then become

$f(0)=S, \quad P(0)=1+S_{f} P^{\prime}(0), \quad P(\infty) \rightarrow 0$.

Using the central difference scheme for derivatives with respect to $\eta$, we can write

$\left(V^{\prime}\right)_{i}=\frac{V_{i+1}-V_{i-1}}{2 \delta \eta}+O\left((\delta \eta)^{2}\right)$

and

$\left(V^{\prime \prime}\right)_{i}=\frac{V_{i+1}-2 V_{i}+V_{i-1}}{(\delta \eta)^{2}}+O\left((\delta \eta)^{2}\right)$,

where $V$ stands for $P$ or $\theta$; i is the grid index in $\eta$-direction with $\eta_{i}=i \times \delta \eta ; \quad i=0,1, \ldots, m \delta \eta$ is the increment along the $\eta$-axis. Newton's linearization method can then be applied to linearize the discretized equations as follows. 
When the values of the unknown functions at the $n$th iteration are known, the corresponding values of these variables at the next iteration are obtained by using the equation

$V_{i}^{n+1}=V_{i}^{n}+\left(\Delta V_{i}\right)^{n}$,

in which $\left(\Delta V_{i}\right)^{n}$ represents the error at the $n$th iteration, $i=0,1,2, \ldots \ldots, n$. It is worthwhile to mention here that the error $\left(\Delta V_{i}\right)^{n}$ at the boundary is zero, because the values of $V_{i}$ at the boundary are known. Using (19) in (15) and dropping the quadratic and higher order terms in $\left(\Delta V_{i}\right)^{n}$, we get a system of block tri-diagonal equations. To solve this tri-diagonal system of equations, we have used the "Tri-diagonal matrix algorithm", usually referred as "Thomas algorithm" (cf. [47-49]). It may be mentioned here that instead of this, one could use Gauss elimination method. But in that case, the number of operations would be $m^{3}$, while in the method that we have employed here, the number of operations is $m$, where $m$ is the number of unknowns. Thus the error committed in our method is much less than that in the method of Gauss elimination. In the process of determination of the distribution of the function $f(\eta)$, the accuracy can be defined as the difference between the calculated values of $f(\eta)$ at two successive operations, say $(n+1)$ th and $n$ th. In the present case, the error $\epsilon$ is equal to

$\epsilon=\left|f^{n+1}(\eta)-f^{n}(\eta)\right|$

and is estimated to be less than $10^{-6}$.

\section{Computation of some theoretical estimates and related discussion}

In order to study the flow and heat transfer of blood in a time-dependent porous narrow blood vessel over a permeable unsteady stretching sheet (as an approximation of a micro-vessel) under slip condition, subject to the action of an external magnetic field, the numerical technique described above has been employed to solve the system of Eqs. (11)-(12), along with the boundary conditions (13) and (14).

In order to achieve the numerical solution, it is necessary to assign some specific values for the dimensionless parameters involved in the analysis presented in Sects. 2 and 3 .

In this section, the computational estimates of different dimensionless parameters involved in the analysis, have been presented in graphical/tabular forms. For performing the computational study, we have made use of the values of various dimensionless parameters (governing the flow problem) that have been reported in scientific literatures by previous experimental/theoretical researchers. Experimental reports reveal that like most other fluids, for human blood the viscosity $\mu$, specific heat at constant pressure $c_{p}$ and the thermal conductivity $k$ are temperature-dependent. On the basis of their experimental studies, Valvano et al. [50] and Chato [51] reported the following data for human blood at a temperature $T=310{ }^{\circ} \mathrm{K}$ :

$\mu=3.2 \times 10^{3} \mathrm{~kg} / \mathrm{ms}, \quad c_{p}=14.65 \mathrm{~J} / \mathrm{kg}^{\circ} \mathrm{K} \quad$ and

$\mathrm{k}=2.2 \times 10^{-3} \mathrm{~J} / \mathrm{ms}^{\circ} \mathrm{K}$

Using these data, we find that the value of the Prandtl number $\operatorname{Pr}=\frac{\mu c_{p}}{k}$ for human blood is 21 .

Keeping this in view and employing the data used by Ishak et al. [45], Hayat et al. [46], Sinha and Misra [49] and Makinde and Osalusi [52], we performed the computation for the following spectrum of values of different parameters:

Unsteadiness parameter $A=0.0,0.3,1.0,3.0,4.0,5.0$

Suction parameter $S=0.0,0.5,1.0,1.5,2.0$

Permeability parameter $k_{3}=0.2,0.3,0.4,0.5$

Velocity slip factor $S_{f}=0.0,0.3,0.6,1.0,1.5,2.0,2.5$, $3.0,4.0$

Thermal slip factor $S_{t}=0.0,0.5,1.0,1.5,2.5$

Prandtl number $P r=21.0,22.0,23.0,24.0,25.0$

Radiation parameter $\mathrm{Nr}=0.0,0.2,1.0,2.0,3.0$

$M^{2}=0.2,0.5,0.7,1.0, M$ being the Hartmann number.

The computational procedure has been executed by taking $\delta \eta=0.0125$ with 321 grid points. The results computed have been reported graphically in Figs. 2, 3, 4, 5, $6,7,8,9,10,11$ and 12 . The results presented in these figures have all been computed by choosing 321 grid points. Figure 13 shows that the results do not change, if we further increase the number of grid points. Figure 14 establishes the validity of our results.

The variation of non-dimensional axial velocity $f$ for different values of Hartmann number $M$, velocity slip factor $S_{f}$, porosity permeability $k_{3}$ and the suction parameter $S$ are shown in Figs. 2, 3, 4 and 5. Figure 2 reveals that axial velocity decreases as Hartmann number $M$ increases. This may be attributed to the fact that an increase in $M$ signifies an enhancement of Lorentz force, thereby reducing the magnitude of the velocity. This figure further indicates that blood velocity in the capillary decreases with increase in distance from the lower wall of the capillary. Figure 3 describes the effects of the velocity-slip factor $S_{f}$ on axial velocity. Clearly velocity in the axial direction reduces when the slip velocity increases. In fact, the quantity $1-f^{\prime}(0)$ increases monotonically with $S_{f}$. So the limiting case (i,e, for large values of $S_{f}$ ) implies that the frictional resistance between the viscous fluid (blood) and the wall is eliminated and stretching of the blood vessel 


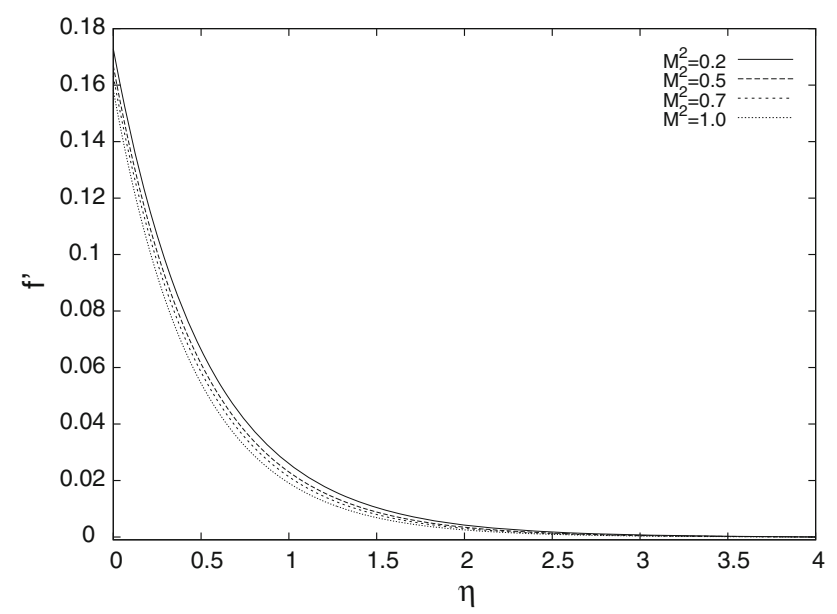

Fig. 2 Velocity distribution for different values of $M^{2}$ with $A=0.3, S_{f}=2.5, S_{t}=1.0, k_{3}=0.4, S=0.5, \operatorname{Pr}=21.0$, and $N r=0.2$

does not significantly add to the motion of the fluid. Figure 4 shows that the axial velocity increases with a rise in permeability. Moreover, for small permeability $\left(k_{3}=0.2\right)$, the axial velocity reduces faster than when the permeability is higher $\left(k_{3}=0.3,0.4,0.5\right)$. Figure 5 gives the distribution of axial velocity for different values of the suction parameter $S$ defined in Eq. (16). The influence of $S$ on axial velocity is similar when compared with Fig. 2, but the reduction in velocity is slightly larger in case of $M$ in comparison to the case of the suction parameter $S$.

Figures $6,7,8,9,10$ and 11 give some characteristic temperature profiles for different values of Hartmann number $(M)$, velocity slip, thermal slip, suction parameter $(S)$, Prandtl number $(\mathrm{Pr})$ and radiation parameter $(\mathrm{Nr})$. The effect of magnetic parameter $(M)$ on temperature profile $\theta$ is shown in Fig. 6. It is noticed that presence of a magnetic field enhances the temperature of the boundary layer of the capillary. This can be attributed to the fact that the introduction of the transverse magnetic field to an electrically conducting fluid gives rise to a resistive type of force known as Lorentz force. This force bears the potential to enhance the temperature of blood. Figures 7 and 8 illustrate the changes that take place in the temperature distribution in blood when the values of velocity-slip factor $\left(S_{f}\right)$ and thermal slip factor $\left(S_{t}\right)$ vary. It is important to observe from these two figures that with a rise in velocity-slip, the temperature at any point in the flow medium increases; however, the temperature reduces when thermal slip enhances. From Fig. 9 which elucidates the influence of the suction parameter $(S)$ on temperature distribution, it is revealed that when the injection/suction velocity increases, the temperature of blood at any point of the flow medium diminishes.

Figure 10 presents the change in the temperature distribution in the boundary layer, when the Prandtl number

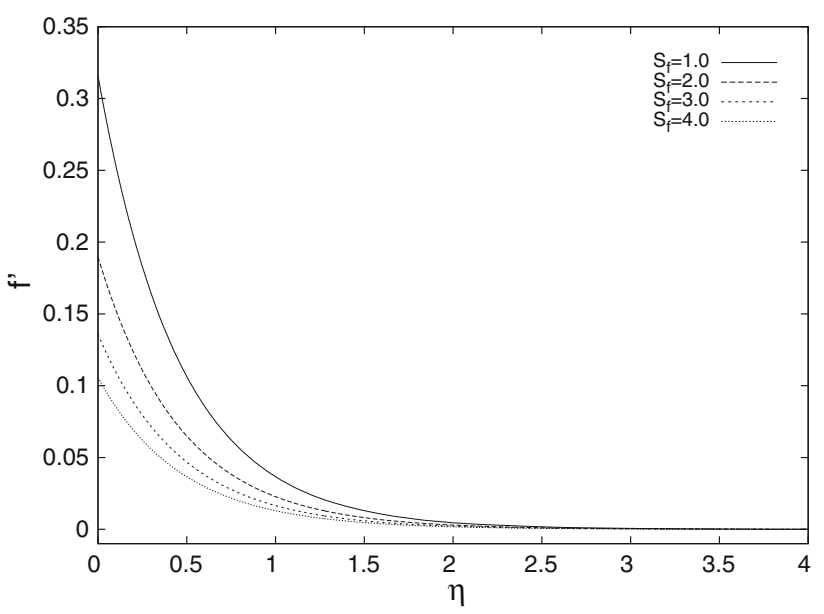

Fig. 3 Velocity distribution for different values of $S_{f}$ with $A=0.3, M^{2}=1.0, S_{t}=1.0, k_{3}=0.4, S=0.5, \quad P r=21.0$, and $\mathrm{Nr}=0.2$

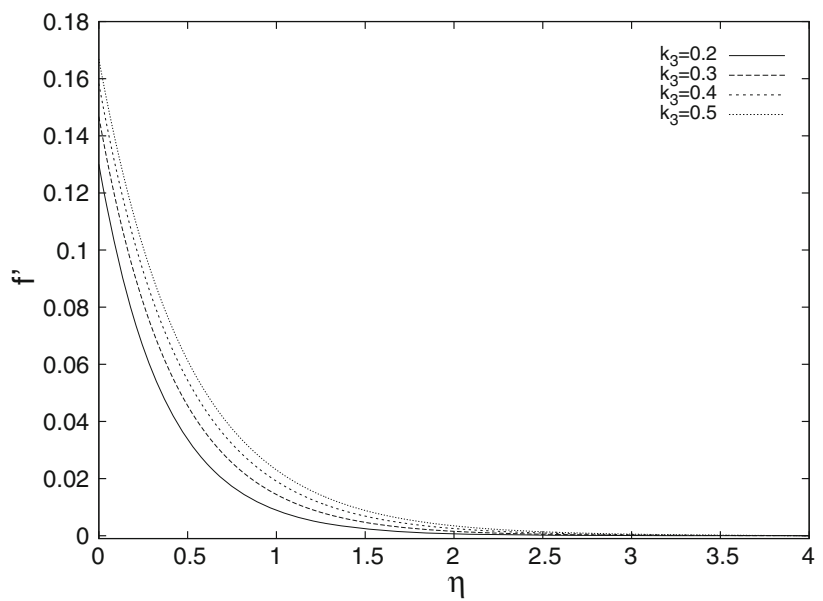

Fig. 4 Velocity distribution for different values of $k_{3}$ with $A=0.3, M^{2}=1.0, S_{t}=1.0, S_{f}=2.5, S=0.5, \quad \operatorname{Pr}=21.0$, and $\mathrm{Nr}=0.2$

$(P r)$ changes gradually. It shows that as the Prandtl number increases, the temperature of the boundary layer diminishes. This may be attributed to the fact that the thermal boundary layer thickness reduces with an increase in Prandtl number. Further, this figure indicates that the temperature gradient at the surface increases with a rise in Prandtl number. This implies that an increase in Prandtl number is accompanied by an enhancement of the heat transfer rate at the wall of the blood vessel. The underlying physics behind this can be described as follows. When blood attains a higher Prandtl number, its thermal conductivity is lowered down and so its heat conduction capacity diminishes. Thereby the thermal boundary layer thickness gets reduced. As a consequence, the heat transfer rate at the vessel wall is increased.

Figure 11 illuminates a very important effect of thermal radiation on the temperature profile. This figure emphasizes 


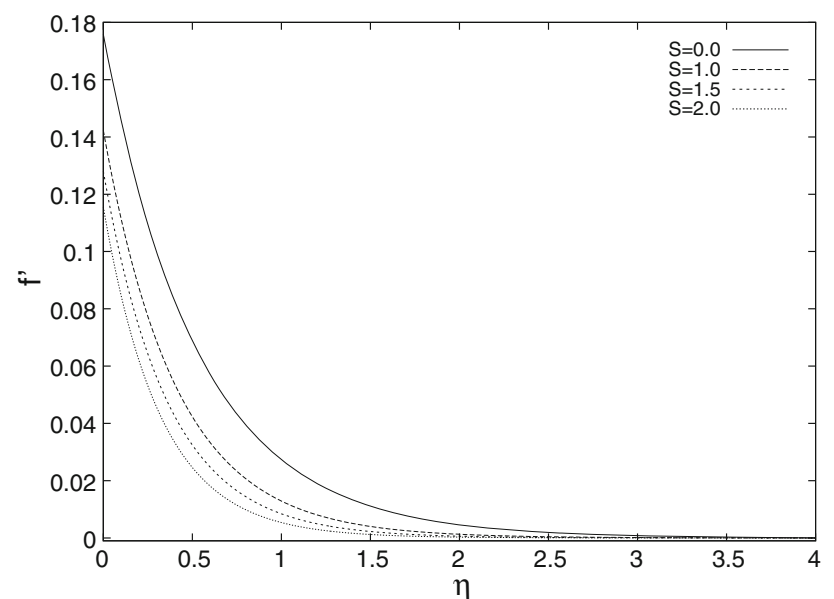

Fig. 5 Velocity distribution for different values of $S$ with $A=0.3, M^{2}=1.0, S_{t}=1.0, S_{f}=2.5, k_{3}=0.4, \operatorname{Pr}=21.0$, and $N r=0.2$

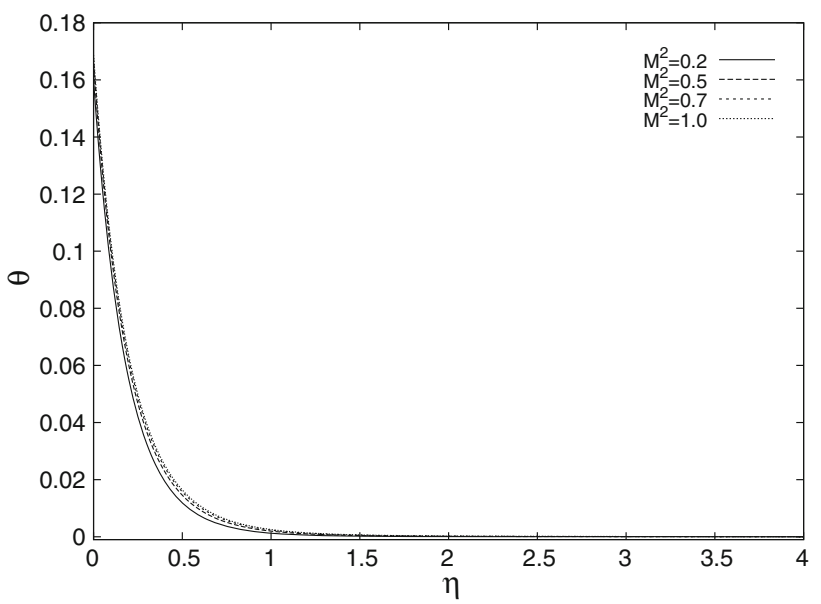

Fig. 6 Temperature distribution for different values of $M^{2}$ with $A=0.3, \quad S=0.5, k_{3}=0.4, \quad S_{f}=2.5, \quad S_{t}=1.0, \quad \operatorname{Pr}=21.0 \quad$ and $\mathrm{Nr}=0.2$

that as thermal radiation increases during blood flow in capillaries, there is a significant rise in the thickness of boundary layer. Thereby the temperature of the boundary layer in enhanced by an appreciable extent.

Of profound interest in problems such as the one under our present consideration, is the skin-friction coefficient denoted by $C_{f}$ and defined by $C_{f}=\frac{\tau_{w}}{\rho U_{w}^{2} / 2}=\frac{1}{2} R e_{x}^{-\frac{1}{2}} f^{\prime \prime}(0), \tau_{w}$ being equal to $\mu\left(\frac{\partial u}{\partial y}\right)_{y=0}$. Figure 12 gives the variation of skin-friction with the Hartmann number for different values of unsteadiness parameter $A$. It is seen that skin-friction increases as the unsteadiness parameter $A$ increases. Another important characteristic that has significant relevance in the present study is the local Nusselt number $N u_{x}$, defined as $\quad N u_{x}=\frac{x q_{w}}{k\left(T_{w}-T_{\infty}\right)}=-\operatorname{Re}_{x}^{-\frac{1}{2}} \theta^{\prime}(0), q_{w} \quad$ being

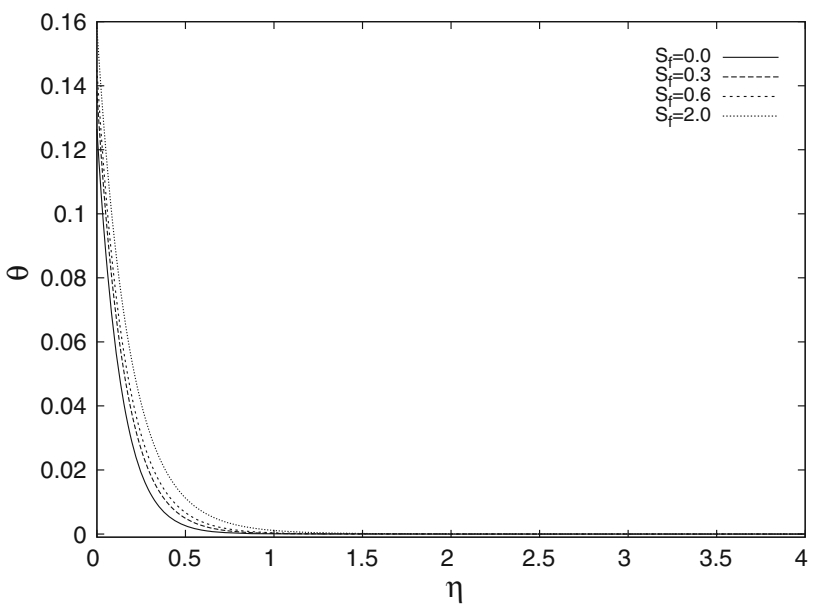

Fig. 7 Temperature distribution for different values of $S_{f}$ with $A=0.3, S=0.5, k_{3}=0.4, M^{2}=0.5, S_{t}=1.0, \quad P r=21.0$ and $\mathrm{Nr}=0.2$

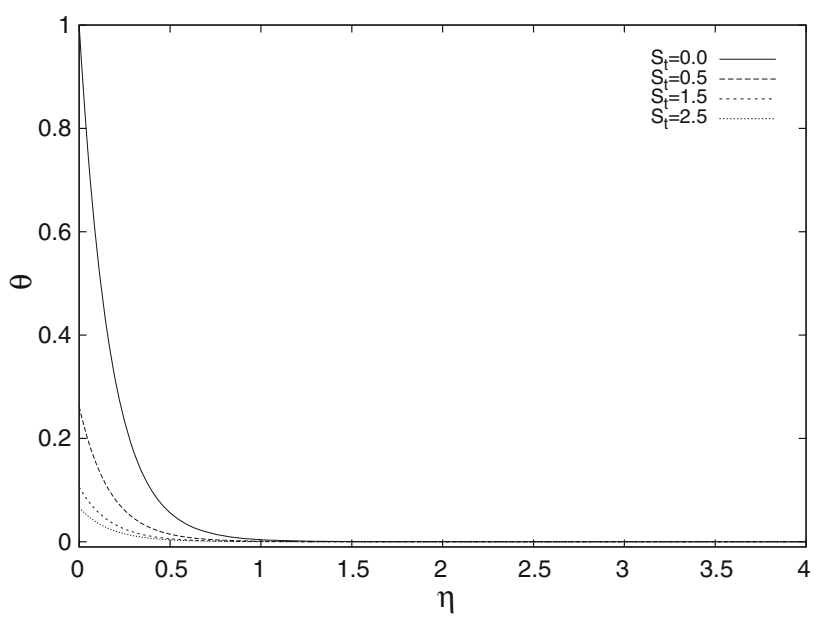

Fig. 8 Temperature distribution for different values of $S_{t}$ with $A=0.3, S=0.5, k_{3}=0.4, M^{2}=0.5, \quad S_{f}=2.5, \quad \operatorname{Pr}=21.0$ and $N r=0.2$

measured by $-k\left(\frac{\partial T}{\partial y}\right)_{y=0}$. The above definition of the local Nusselt number shows that its variation depends on the variation of the factor $\theta^{\prime}(0)$, the values of which on the stretching wall, as per our computational study are presented below in tabular form. The tabulated values correspond to the particular case when $k_{3}=0.4, S=0.5$, $S_{f}=2.5$ and $S_{t}=1.0$.

The computed values displayed in Table 1 indicate that an increase in local Nusselt number, when the value of any of the parameters $A, M$ or $N r$ increases, the local Nusselt number will diminish.

In order to perform grid independent test for the choice of 321 grid points, we repeated the computational procedure by considering a number of mesh sizes by altering the values of $\delta \eta$. Figure 13 shows that the plots for the velocity distribution for $\delta \eta=0.008$ (mesh size $=501$ ) and for 


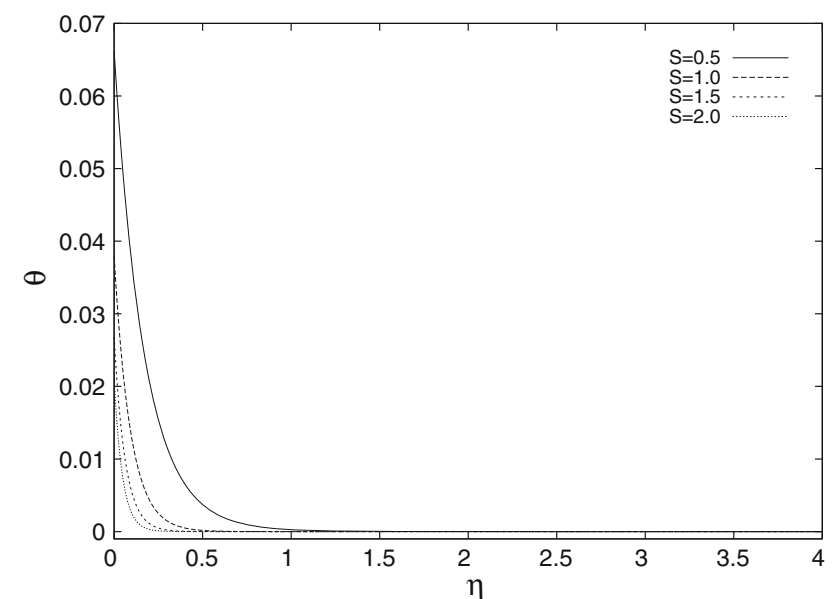

Fig. 9 Temperature distribution for different values of $S$ with $A=0.3, S_{t}=1.0, k_{3}=0.4, M^{2}=0.5, S_{f}=2.5, \quad \operatorname{Pr}=21.0$ and $\mathrm{Nr}=0.2$

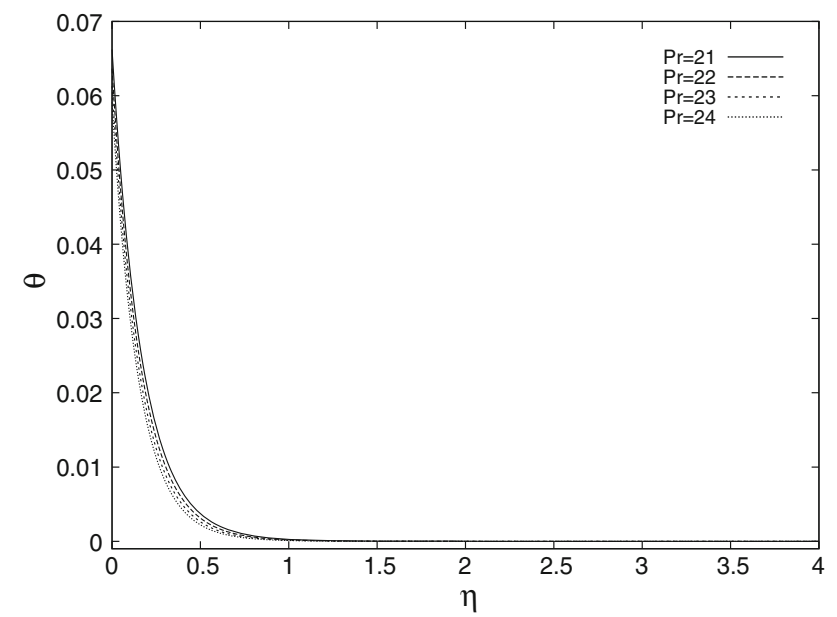

Fig. 10 Temperature distribution for different values of $P r$ with $A=0.3, S_{t}=1.0, k_{3}=0.4, M^{2}=0.5, S_{f}=2.5, S=0.5 \quad$ and $N r=0.2$

$\delta \eta=0.01($ mesh size $=401)$ coincide with the plot corresponding to $\delta \eta=0.0125$, that is, to a mesh size of 321 . It has also been ascertained that for any mesh size less than 321 , the results are inaccurate.

In order to validate the results of our study, which we obtained by employing our numerical procedure, we have made an attempt to compare with the numerical results reported by Ishak et al. [45], which they obtained by using their analytical solution. For the purpose of a meaningful comparison, we had to bring our problem to the same platform as that of [45], by setting $M=0.0, S_{f}=0.0$, $A=0.0, S=1.0$. Moreover, for making the comparison, we considered in our study a large value of the permeability parameter (taking $k_{3}=100.0$ ).

The analytical solution for $f(\eta)$ presented by Ishak et al. [45] is given by $f(\eta)=\zeta-\frac{1}{\zeta} e^{-\eta \zeta}$, so that $f^{\prime}(\eta)=e^{-\eta \zeta}$

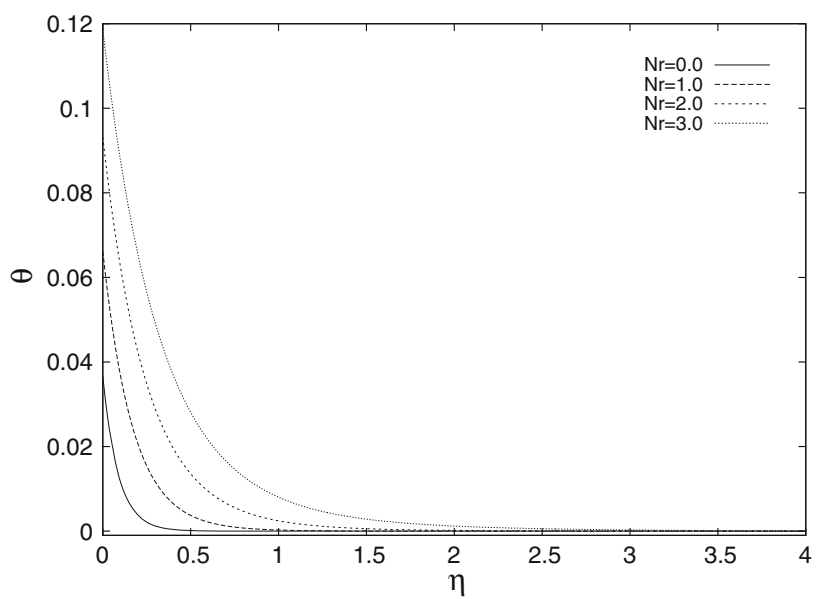

Fig. 11 Temperature distribution for different values of $\mathrm{Nr}$ with $A=0.3, S_{t}=1.0, k_{3}=0.4, M^{2}=0.5, S_{f}=2.5, \quad S=0.5 \quad$ and $\operatorname{Pr}=21.0$

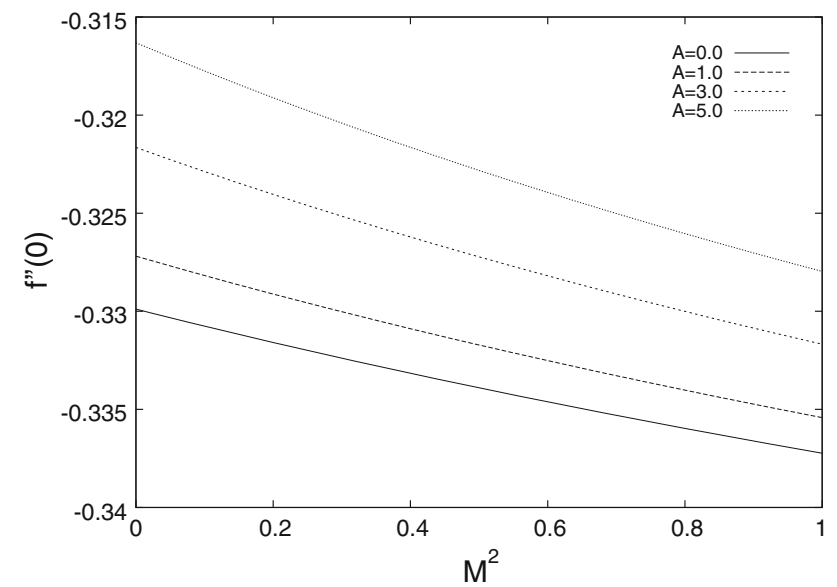

Fig. 12 Variation of skin-friction with $M$ for different values of unsteadiness parameter $A$ with $k_{3}=0.4, S=0.5$ and $S_{f}=2.5$

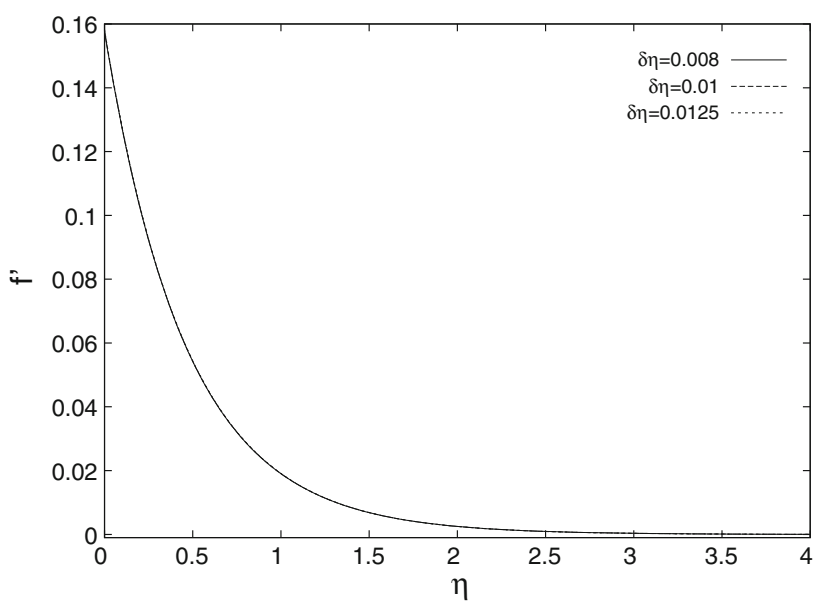

Fig. 13 Velocity distribution for different mesh sizes 


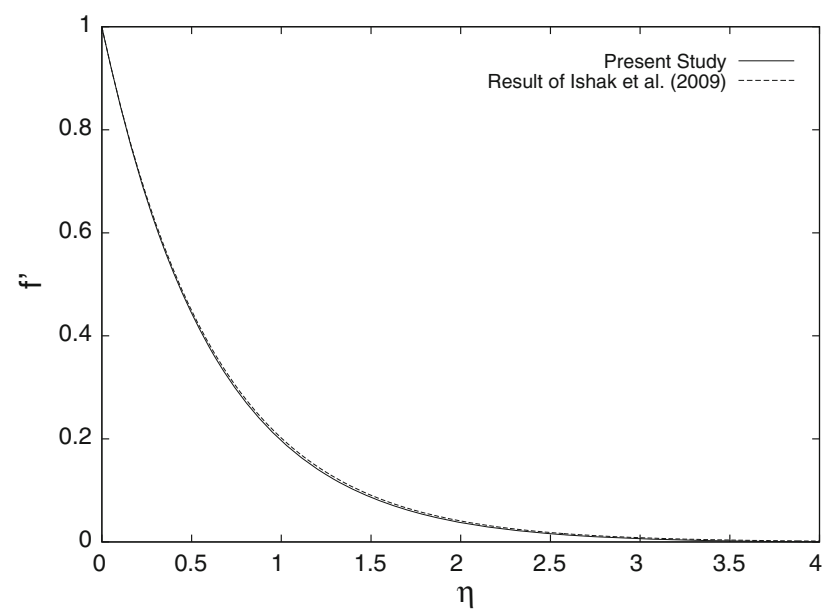

Fig. 14 Velocity distribution for $M=0.0, S_{f}=0.0, \quad A=0.0$, $S=1.0$ and $k_{3}=100.0$ (comparison of the results of the present study with the numerical results of Ishak et al. [45] on the basis of their analytical solution)

Table 1 Numerical values of $\theta^{\prime}(0)$ for different values of unsteadiness parameter $(A)$, Hartmann number $(M)$, Prandtl number $(P r)$ and radiation parameter $(\mathrm{Nr})$

\begin{tabular}{lllll}
\hline$A$ & $M^{2}$ & $N r$ & $P r$ & $-\theta^{\prime}(0)$ \\
\hline 3.0 & 0.5 & 1.0 & 21.0 & 0.4659137 \\
3.0 & 0.5 & 1.0 & 25.0 & 0.5335517 \\
3.0 & 0.5 & 2.0 & 21.0 & 0.3294789 \\
3.0 & 1.0 & 1.0 & 21.0 & 0.4242029 \\
4.0 & 0.5 & 1.0 & 21.0 & 0.3904817 \\
\hline
\end{tabular}

In their analysis, $f_{0}=\zeta-\frac{1}{\zeta}$ is the suction/injection parameter, while in the analysis of our model, $S$ stands for the injection/suction parameter.

The results of our study for the particular case described above and those of [45] have been presented side by side in Fig. 14. It may be noted that our results are in excellent agreement with those reported by Ishak et al. [45].

\section{Summary and conclusion}

The mathematical model formulated and analyzed in the study has been motivated towards investigating the effect of thermal radiation on the flow as well as heat transfer in a narrow permeable blood vessel, when the system is subject to the action of an external magnetic field. The study is particularly applicable to a situation, where the lumen of the blood vessel has turned into a porous medium due to the deposition of cholesterol, fatty substances etc., our target being to study the flow pattern and heat transfer in the blood vessel when it is in a pathological state. The blood vessel is considered to execute an unsteady stretching motion. The erythrocyte slip at the vessel wall and the thermal slip have been duly accounted for. All these considerations have made the study quite close to a real situation.

The study bears the potential to explore some important information regarding the complex flow behaviour of blood in situations, where all the eight physical parameters $M, A, S, k_{3}, S_{f}, S_{t}, \operatorname{Pr}$ and $N r$ play prominent roles on the hemodynamical flow and heat transfer in arterioles. The numerical estimates presented here elucidate the effects of the said parameters. These may be summarized as follows:

1. The velocity of blood can be controlled by suitably regulating the intensity of the external magnetic field.

2. Thermal radiation bears the potential to bring about a significant change in the temperature field of the boundary layer. With a rise in thermal radiation, the thermal boundary layer thickness increases by an appreciable extent.

3. An increase in erythrocyte-slip at the capillary wall leads to an increase in the temperature of the boundary layer, while an increase in thermal slip reduces the temperature field.

4. When the injection/suction velocity increases, the velocity and temperature of blood in the capillary both diminish.

5. The temperature of blood inside the boundary layer also reduces, if the Prandtl number increases.

6. The velocity of blood along the axis of the capillary increases with a rise in permeability.

In order to treat the patients more accurately with an aim to get better results in thermal therapy for relieving pain, the theoretical estimates presented here will be useful. Moreover, the study gives an understanding of the thermal processes that take place during blood flow in micro-vessels of the arterial tree. The study will also be of sufficient interest to clinicians who are engaged in the treatment of cancers and tumors by using the method of electromagnetic hyperthermia. This is because this technique involves overheating the affected tissues, usually around $42{ }^{\circ} \mathrm{C}$. This study will also find applications in estimating the electromagnetic radiation when a human being has to work in radiation fields. Owing to the reason that the study takes into account heat transfer, it will find application in performing heat-dose sensitivity tests, which are required for using the method of hyperthermia in an appropriate manner.

Since the study takes into account velocity-slip and also since blood velocity in micro-vessels is low, it bears the potential to furnish some additional information regarding the causes and development of arterial diseases, such as atherosclerosis. It is worthwhile to mention here the conjecture made by Caro et al. [53] that arterial diseases, like 
atherosclerosis occur mostly in regions, where blood velocity is low. Due to similar reasons, the study will find applications in surgical operations, where it is necessary to reduce the blood flow rate, since this study clearly reveals that reduction of the volumetric flow rate of blood can be accomplished by exposing the system to a magnetic field. Further, the present study will be useful in assessing the accuracy of future experimental/theoretical studies of more complex nature, that may involve greater number of physical parameters.

Acknowledgment The authors are thankful to Professor Andrea Luke, Editor-in-Chief, HMT and all the three anonymous reviewers for their kind words of appreciation regarding the quality of the manuscript and also for their kind suggestions for the improvement. One of the authors, Prof. J. C. Misra wishes to express his deep sense of gratitude to Prof. (Dr.) Manoj Ranjan Nayak, President of Siksha O Anusandhan Univesity, Bhubaneswar, India for the necessary help and kind encouragement in his current research activities.

Open Access This article is distributed under the terms of the Creative Commons Attribution License which permits any use, distribution, and reproduction in any medium, provided the original author(s) and the source are credited.

\section{References}

1. Kobu Y (1999) Effects of infrared radiation on intraosseous blood flow and oxygen tension in rat tibia. Kobe J Med Sci 45:27-39

2. Inoue S, Kobaya M (1989) Biological activities caused by farinfrared radiation. Int J Biometeorol 33:145-150

3. Nishimoto C, Ishiura Y, Kuniasu K, Koga T (2006) Effects of ultrasonic radiation on cutaneous blood flow in the paw of decerebrated rats. Kawasaki J. Med Welfare 12:13-18

4. Prakash J, Makinde OD (2011) Radiative heat transfer to blood flow through a stenotic artery in the presence of magnetic field. Lat Am Appl Res 41:273-277

5. He Y, Shirazaki M, Liu H, Himeno R, Sun Z (2006) A numerical coupling model to analyze the blood flow, temperature, and oxygen transport in human breast tumor under laser irradiation. Comp Biol Med 36:1336-1350

6. Ogulu A, Bestman AR (1994) Blood flow in a curved pipe with radiative heat transfer. Acta Physica Hungarica 74:189-201

7. Shah J, dos Santos I, Hammerich D, Valvano JW (2005) Instrument to measure the heat convection coefficient on the endothelial surface of arteries and veins. Med Biol Eng Comput 43:522-527

8. Craciunescu OI, Clegg ST (2001) Pulsatile blood flow effects on temperature distribution and transfer in rigid vessels. J Biomech Eng 123:500-505

9. Horng TL, Lin WL, Liauh CT, Shih TC (2007) Effects of pulsatile blood flow in large vessels on thermal dose distribution during thermal therapy. Med Phys 34:1312-1320

10. Shrivastava D, Roemer RB (2005) An analytical study of "poisson conduction shape factors' for two thermally significant vessels in a fine, heated tissue. Phys Med Biol 50:3627-3641

11. Szasz A (2007) Hyperthermia, a modality in the wings. J Cancer Res Ther 3:56-66

12. Misra JC, Sinha A, Shit GC (2010) Flow of a biomagnetic viscoelastic fluid: application to estimation of blood flow in arteries during electromagnetic hyperthermia, a therapeutic procedure for cancer treatment. Appl Math Mech Eng Educ. 31:1405-1420

13. Abe M, Hiraoka H (1985) Localized hyperthermia and radiation in cancer therapy. Int J Radiat Biol Relat Stud Thys Chen Med 47:347-359

14. Molls M (1992) Hyperthermia-the actual role in radiation oncology and future prospects, part I. Strahlen Onkol 168:183-190

15. Chen ZP, Roemer RB (1992) The effects of large blood vessels on temperature distributions during simulated hyperthermia. ASME J Biomech Eng 114:473-481

16. Overgaard J (1989) The current and potential role of hyperthermia in radiotherapy. Int J Radiat Oncol Biol Phys 16:535-549

17. Feldmann HJ, Seegenschmiedt MH, Molls M (1995) Hyperthermia-its actual role in radiation oncology, part III: clinical rationale and results in deep seated tumors. Strahlenther Onkol 171:251-264

18. Habash RWY, Bansal R, Krewski D, Alhafid HT (2007) Thermal Therapy, Part 3: ablation techniques. Crit. Rev. Biomed. Engg. 35:37-121

19. Deng ZS, Liu J, Wang HW (2008) Disclosure of the significant thermal effects of large blood vessels during cryosurgery through infrared temperature mapping. Int J Therm Sci 47:530-545

20. Caro CG, Pedley TJ, Schroter RC (1978) The mechanics of circulation. Oxford University Press, Oxford

21. Oka S, Murata T (1970) A theoretical study of the flow of blood in a capillary with permeable wall. Jpn J Appl Phys 9:345-352

22. Pallat PJ, Sackin H, Tanner RT (1974) A hydrodynamic model of a permeable tubule. J Theor Biol 44:287-303

23. Vankan WJ, Huyghe JM, Drost MR, Janssen JD, Huson A (1997) A finite element mixture model for hierarchical porous media. Int J Numer Math Eng 40:197-210

24. Dash RK, Mehta KN, Jayaraman G (1996) Casson fluid flow in a pipe filled with homogeneous porous medium. Int J Eng Sci 34:1146-1156

25. Preziosi L, Farina A (2002) On Darcy's law for growing porous media. Int J Non-linear Mech 37:485-491

26. Chen IH (1985) Analysis of an intensive magnetic field on blood flow: part 2. J Bioelectr 4:55-61

27. Tzirtzilakis EE, Tanoudis GB (2003) Numerical study of biomagnetic fluid flow over a stretching sheet with heat transfer. Int J Numer Methods Heat Fluid Flow 13:830-848

28. Wilson DH (1974) Comparison of short wave diathermy and pulsed electromagnetic energy in treatment of soft tissue injuries. Physiotherapy 60:309-310

29. Varcaccio G, Carriero C, Loizzo MR (1995) Analgesic properties of electromagnetic field therapy in patients with chronic pelvic pain. Clin Exp Obstet Gynecol 22:350-354

30. Sisken BF, Kanje M, Lundbirg G (1990) Pulsed electromagnetic fields stimulate nerve regeneration in vitro and in vivo. Restor Neurol Neurosci 1:303-309

31. Kort J, Ito H, Bassett CAL (1980) Effects of pulsing electromagnetic fields on peripheral nerve regeneration. Orthop Trans 4:238-239

32. Mooney V (1990) A randomized double-blind prospective study of the efficacy of pulsed electromagnetic fields for interbody lumbar fusions. Spine 15:708-712

33. Sharrard WJW (1990) Double blind trial of pulsed electromagnetic fields for delayed union of tibial fractures. J Bone Joint Surg Br 72:347-355

34. Liu IC (2004) Flow and heat transfer of an electrically conducting fluid of second grade over a stretching sheet subject to a transverse magnetic field. Int J Heat Mass Transf 47:4427-4437

35. Sajid M, Hayat $T$ (2008) Influence of thermal radiation on the boundary layer flow due to an exponentially stretching sheet. Int Commun Heat Mass Transf 35:347-356 
36. Beavers GS, Joseph DD (1967) Boundary conditions at a natural permeable wall. J Fluid Mech 30:197-207

37. Nubar Y (1971) Blood flow, slip and viscometry. J Biophys 11:252-264

38. Misra JC, Patra MK, Misra SC (1993) A non-Newtonian fluid model for blood flow through arteries under stenotic conditions. J Biomech 26:1129-1141

39. Misra JC, Shit GC (2007) Role of slip velocity in blood flow through stenosed arteries: A non-Newtonian model. J Mech Med Biol 7:337-353

40. Pal B, Misra JC, Gupta AS (1996) Steady hydromagnetic flow in a slowly varying channel. Proc Natl Inst Sci Ind Part A 66:247-262

41. Misra JC, Shit GC (2009) Biomagnetic viscoelastic fluid flow over a stretching sheet. Appl Math Comput 210:350-361

42. Misra JC, Shit GC, Rath HJ (2008) Flow and heat transfer of a MHD viscoelastic fluid in a channel with stretching walls: some applications to Hemodynamics. Comput Fluids 37:1-11

43. Raptis A (1998) Radiation and free convection flow through a porous medium. Int Commun Heat Mass Transf 25:289-295

44. Misra JC, Sinha A, Shit GC (2011) A numerical model for the magnetohydrodynamic flow of blood in a porous channel. J Mech Med Biol 11:547-562

45. Ishak A, Nazar R, Pop I (2009) Heat transfer over an unsteady stretching permeable surface with prescribed wall temperature. Nonlinear Anal Real World Appl 10:2909-2913
46. Hayat T, Qasim M, Mesloub S (2010) MHD flow and heat transfer over permeable stretching sheet with slip conditions. Int J Numer Methods Fliuds 66: 2909-2913 doi:10.1002/fld.2294

47. Conte SD, Deboor C (1992) Elementary numerical analysis. McGraw-Hill, New York

48. Misra JC, Sinha A, Shit GC (2010) Flow of a biomagnetic viscoelastic fluid: application to estimation of blood flow in arteries during electromagnetic hyperthermia, a therapeutic procedure for cancer treatment. App. Math Mech Engl Educ 31:1405-1420

49. Sinha A, Misra JC (2012) Numerical study of flow and heat transfer during oscillatory blood flow in diseased arteries in presence of magnetic fields. Appl Math Mech Engl Educ 33:649-662

50. Valvano JW, Nho S, Anderson GT (1994) Analysis of the Weinbaum-Jiji model of blood flow in the canine kidney cortex for self-heated thermistorsrom. J Biomech Eng 116:201-207

51. Chato JC (1980) Heat tranfer to blood vessels. J Biomech Eng 102:110-118

52. Makinde OD, Osalusi E (2006) MHD steady flow in a channel with slip at permeable boundaries. Rom J Phys 51:319-328

53. Caro CG, Fitz-Gerald JM, Schroeter RC (1971) Atheroma and arterial wall shear stress observation, correlation and proposal of a shear dependent mass transfer mechanism for atherogenesis. Proceed R Soc Lond Ser B 177:109-159 